\title{
AIDS: a bill of rights for the surgical team?
}

\author{
H A F DUDLEY, A SIM
}

Surgeons have not been much involved with decision making over AIDS policy. In our institution AIDS has largely been regarded as a "medical" condition with occasional problems for which the surgeon can be called in. As more patients enter the florid clinical phase of the disease and as life may be prolonged by the use of agents that prevent viral expression, surgical exposure to the condition will inevitably increase for two reasons: firstly, because AIDS does give rise to a burden of surgery in its own right; secondly, because patients with human immunodeficiency virus (HIV) infection can suffer from other ills just like all of us. For example, at this moment we have in our ward at this hospital: one patient who has undergone laparotomy for a mixed lesion of the terminal ileum, comprising cryptosporidiosis, cytomegalovirus infection, and Kaposi's sarcoma; one patient who has required a lymph node biopsy and a laparotomy for a high grade lymphoma; and one patient with confirmed AIDS undergoing non-operative treatment for adhesive obstruction. We operate for other conditions about three times a month. Though our experience is for the moment exceptional, national policy is to disseminate care, and other surgeons will undoubtedly be seeing similar patients over the next decade.

The risk of infection for surgeons remains, as far as is currently known, statistically extremely small but is different from and probably greater than that of the non-operating clinician or the laboratory worker. The clinician controls the risk by limiting invasive contacts with the patient to venepuncture and perhaps controlled sampling of other secretions. The laboratory worker has a code of laboratory practice designed to give him or her maximum protection-though as history relates for other infectious disease some risk may still persist. By contrast, surgeons work in a less

Academic Surgical Unit, St Mary's Hospital, London W2 1NY H A F DUDLEY, CHM, FRCS, professor

A SIM, MS, FRCS, assistant director

Correspondence to: Professor Dudley. controlled environment. They have an occupational hazard of self injury which reaches at least one in every 20 operations ${ }^{1}$; they cannot always avoid operating when they have a small open lesion on their hands; and their level of exposure to blood and other secretions is much higher than any other group. It is somewhat surprising that so far very few surgeons are known to have become infected, but this observation does not mean we should be complacent. We are aware of anecdotal information that further instances of seropositivity in surgeons not at high risk from their personal behaviour are about to be recorded. It is fair also to record that our expectations for freedom from occupational hazard in our work have risen. As a house officer and registrar, one of us, along with his contemporaries, had to be fatalistic about exposure to tuberculosis (an almost ritualistic illness among thoracic surgical staff in the 'forties and early 'fifties) and hepatitis, but this would now be intolerable.

A surgeon or any member of his or her team will not wish to place the HIV positive patient at risk from inadequate care. As with the patient's right to treatment, however, we believe that the team should also have some rights whether or not they choose to exercise them. Among these might be the following:

(1) To insist on standards in the operating room that are adequate to give the best protection of the surgical team from the risk of cross infection. It is now policy in our district to consider any patient as being infected. Carried to its logical conclusion we are thus entitled to regard every patient who undergoes surgery as requiring "hepatitis level" precautions. This would need, at a rough estimate, a doubling of available theatre use at a time when we have woefully inadequate funding. As we have said elsewhere ${ }^{2}$ resources to develop new, less traumatic or dangerous techniques are also needed as well as resources to purchase new equipment which will reduce the use of "sharps." We are glad to say that at least we have been allotted ultrasound equipment for that purpose.

(2) To reserve the right not to operate on patients known to have HIV or who are categorised as at high risk when circumstances might be unfavourable for any member of the team, always provided that an alternative surgeon is available.

(3) To have the opportunity if they so wish formally to disclaim high risk in themselves, though the mechanism for this will be 
complicated. We make this point because the history of the AIDS epidemic suggests that there is still an automatic tendency to stigmatise the infected person as of necessity belonging to a "high risk" group.

(4) To have blood withdrawn at regular intervals for storage against a future need to establish HIV state. This facility already exists in our district, and the matter is very carefully arranged to ensure confidentiality but we are not aware of any national policy.

(5) To have blood withdrawn and possibly analysed at the time of and subsequent to a specific incident in which virus transmission was a possibility. Because of the definition adopted in our district this would in theory embrace all operative injuries.

(6) To have an HIV assay at their own behest. Again, with suitable safeguards, this is possible in our district.

(7) To have the "signing on" for work and the insurance situation debated at greater length. In particular, is it likely to become a condition of service that the members of the surgical team are initially and perhaps intermittently tested for their HIV state? Are those in contact with patients with AIDS likely to have a loading on their insurance premiums?
(8) To request - and in our view to insist-on testing for HIV of a patient involved in an injury to a surgeon. Reassurance from a negative assay for HIV antibodies in the patient does not, because of the incubation period, carry certainty, but at least it would have a confidence building effect to which the surgical "victim" is entitled.

These proposals will undoubtedly arouse controversy and by some, away from the front line, be regarded as inappropriate surgical panic. It has, however, characterised the AIDS "epidemic" that reactions to events have been post hoc rather than propter hoc. ${ }^{3}$ It is wise to discuss the matter now against a background of suggestions, some of which may be less soundly based than others, but all of which should be debated. By so doing we may also be readier for the next epidemic, whatever this may be.

\section{References}

1 Hussain SA, Latif ABA, Choudray AAAA. Risk to surgeons: a survey of accidental injuries during operations. Brf Surg 1988;75:314-6.

2 Sim AJW, Dudley HF. Surgeons and HIV. Br Med $\mathcal{f}$ 1988;296:80. 3 Shilts R. The band played on. New York: St Martin's Press, 1987.

\title{
Contemporary Themes
}

\section{Accidents in the home among children under 5: ethnic differences or social disadvantage?}

\author{
RAFI ALWASH，MARK MCCARTHY
}

\begin{abstract}
Accidents in the home to children under 5 in a multiracial population with a high level of social disadvantage were studied by interviewing at home the parents of $\mathbf{4 0 2}$ children attending the accident department of a west London hospital during one year. The parents' country of birth, whether they were employed, and their housing conditions were recorded using the definitions of the 1981 census. Four ethnic groups (British (183 children), Asian (127), Caribbean (61), and other (31)) were identified. Though attendance rates based on the populations of electoral wards at the census and standardised for distance from the hospital showed no significant differences among the ethnic groups, there was a strong gradient by social class and strong associations with unemployment of the mother (although not of the father), overcrowding, and tenure of housing.

Social disadvantage seems to be more important than ethnicity as a determinant of accidents to children in the home.
\end{abstract}

\footnotetext{
Department of Community Medicine, University College London, London WC1E 6EA

RAFI ALWASH, MB, PHD, research fellow

MARK MCCARTHY, FFCM, MRCP, senior lecturer

Correspondence to: Dr McCarthy.
}

\section{Introduction}

Accidents are the commonest cause of death among children under 15 and the second commonest, after congenital anomalies, among children under 5. Fatal accidents to older children are most often caused by motor vehicles, but in children under 5 they commonly occur in the home.' About half a million children under 5 attend accident and emergency departments each year for injuries resulting from accidents in the home (Department of Trade and Industry, home accident surveillance system, personal communication).

Little is known about accidents to children in ethnic minorities in Britain. The number of deaths so far has been small. Ethnicity is not routinely recorded in National Health Service data but was considered in two studies of attendances at accident departments. In Preston Ahamed estimated that the rate of hospital attendance after accidents in the home was similar among Asian children under 5 and among all children under 5 in the town, whereas in Bradford Learmonth reported that there were more burns and scalds than expected in city wards with high populations from new Commonwealth countries. ${ }^{23}$ Both studies failed to take account of the gradient of use that occurs with distance from the accident department; Learmonth's study used a correlation matrix rather than calculating rates specific to wards; and both studies used different classifications of ethnicity from census definitions.

Accidental deaths of children show a gradient according to the Registrar General's index of social class. ${ }^{4}$ Studies of the distribution of non-fatal injuries by social class, however, have yielded conflicting results. Brown and Davidson's study of accidents in children up to the age of 15, MacQueen's two year prospective study of accidents in the home in Aberdeen, and Martin's investigation of 